\title{
Contracture Caused by Sodium Removal in the Pregnant Rat Myometrium
}

\author{
Katsuji Matsuzawa, Tetsuo Masahashi, Masamichi Kinira, \\ and Tadao Tomita* \\ Department of Obstetrics and Gynaecology, and * Department of Physiology, \\ School of Medicine, Nagoya University, Nagoya, 466 Japan
}

\begin{abstract}
The contracture produced by removal of the external $\mathrm{Na}$ was studied in the pregnant rat myometrium and this was compared with that of the non-pregnant myometrium. The $\mathrm{Na}$-free contracture was small compared with spontaneous phasic contractions in the circular muscle of non-pregnant rat, but the contracture increased during pregnancy. On the other hand, in the longitudinal muscle, the contracture was large in both non-pregnant and pregnant myometria. The change in circular muscle did not seem to result from an increased sensitivity to the external Ca. Since the contracture induced by $40 \mathrm{~mm} \mathrm{~K}$ had properties similar to those of the $\mathrm{Na}$-free contracture, it is likely that weak tension development in nonpregnant circular muscle is either due to a limited $\mathrm{Ca}$ influx or due to a low efficiency of the excitation-contraction coupling for the sustained contraction compared with the phasic contraction. A small contraction which was independent of the external $\mathrm{Ca}$ was observed in $\mathrm{Na}$-free solution in the both layers of non-pregnant myometrium, but this was not observed in the longitudinal muscle of pregnant myometrium.
\end{abstract}

Key words: smooth muscle, myometrium, Na-removal, contracture.

Reduction of the external $\mathrm{Na}$ concentration is known to produce an increase in tension in the longitudinal muscle of the pregnant myometrium of the rat (MARShall, 1963) and the mouse (OSA, 1971, 1973). In the previous paper (MASAhashi and Tomita, 1983), we compared the mechanical responses caused by Na-removal in the longitudinal muscle with those of the circular muscles in the nonpregnant rat myometrium. It has been shown that the response to Na-removal is much larger in the longitudinal than the circular muscle, and that there are two components in the tension development in $\mathrm{Na}$-free solution, one being dependent on, and the other being independent of, extracellular $\mathrm{Ca}$.

The Ca-dependent component of contraction produced on Na-removal is likely

Received for publication September 24, 1986 
to be due to an increase in Ca conductance of the plasma membrane, because it is readily abolished by removing the external Ca (Osa, 1971; MASAHASHI and TOMITA, 1983). Involvement of a $\mathrm{Na}-\mathrm{Ca}$ exchange mechanism has also been suggested (OsA, 1973; Angles D'Auriac and Worcel, 1976; MA and Bose, 1977), but in the nonpregnant rat myometrium, this mechanism seems to contribute to only the early transient phase of the response (MASAHASHI and TOMITA, 1983).

In the myometrium, it is well known that the electrical and mechanical activities change remarkably depending on the stage of oestrous cycle and also during pregnancy (OsA and FuJINO, 1978; KISHIKAWA, 1981). No clear difference has been found in the effect of Na-removal during oestrous cycle in the rat (MASAHASHI and Tomita, 1983), but the difference between non-pregnant and pregnant myometria has not been investigated. Therefore, in the present experiments this problem was examined. It was found that the tension development in the circular muscle produced by Na-removal became much larger during late pregnancy, but that the change in the longitudinal muscle was rather small.

\section{METHODS}

The preparation of muscle strips and the experimental procedure were the same as those described in the previous paper (MASAHASHI and ToMITA, 1983). Nonpregnant (oestrous stage) and pregnant (the 12th-20th day of pregnancy) Wistar rats were used for experiments. The rat at prooestrus was left overnight to mate, and when the presence of sperm was confirmed in the smear test the following morning, the day was counted as the first day of pregnancy. Strips, about 1-mm wide and 7$\mathrm{mm}$ long, were cut in the longitudinal or the circular direction of the uterine horn, after the rats were stunned and bled. Following careful removal of the endometrium, the circular and longitudinal muscle layers were separated and trimmed to the same size. The longitudinal and circular muscle strips were mounted in the same organ bath ( $1 \mathrm{ml}$ in capacity) and the tension was isometrically recorded using a strain gauge and a potentiometric pen-recorder. The solution, kept at $35^{\circ} \mathrm{C}$, flowed at a constant rate of $1.5 \mathrm{ml} / \mathrm{min}$ through the organ bath.

The preparations were equilibrated in normal solution for at least $1 \mathrm{~h}$ and the resting tension was adjusted to $0.05 \mathrm{~g}$ in $\mathrm{Ca}-$ free solution before starting the experiment. Normal solution had the following composition (mM): $\mathrm{NaCl} 121.5$, $\mathrm{KHCO}_{3} 5.9, \mathrm{CaCl}_{2} 2.4, \mathrm{MgCl}_{2} 1.2$, glucose 11.8 , tris hydroxymethyl aminomethane (Tris) $15.3, \mathrm{pH}$ was adjusted to 7.4 at $35^{\circ} \mathrm{C}$ by $\mathrm{HCl}$. When the concentration of $\mathrm{NaCl}$ or $\mathrm{CaCl}_{2}$ was changed, they were usually replaced with Tris on an equiosmolar basis. In some experiments, choline chloride was used as a $\mathrm{Na}$ substitute in the presence of atropine $(10 \mu \mathrm{M})$, but there was no fundmantal difference between choline and Tris. In Ca-free solution, $0.5 \mathrm{~mm}$ ethylene-glycolether tetraacetic acid (EGTA) was added.

The tonic contraction produced by Na-removal was compared with the spontaneous phasic contractions or phasic contractions induced by oxytocin (10 


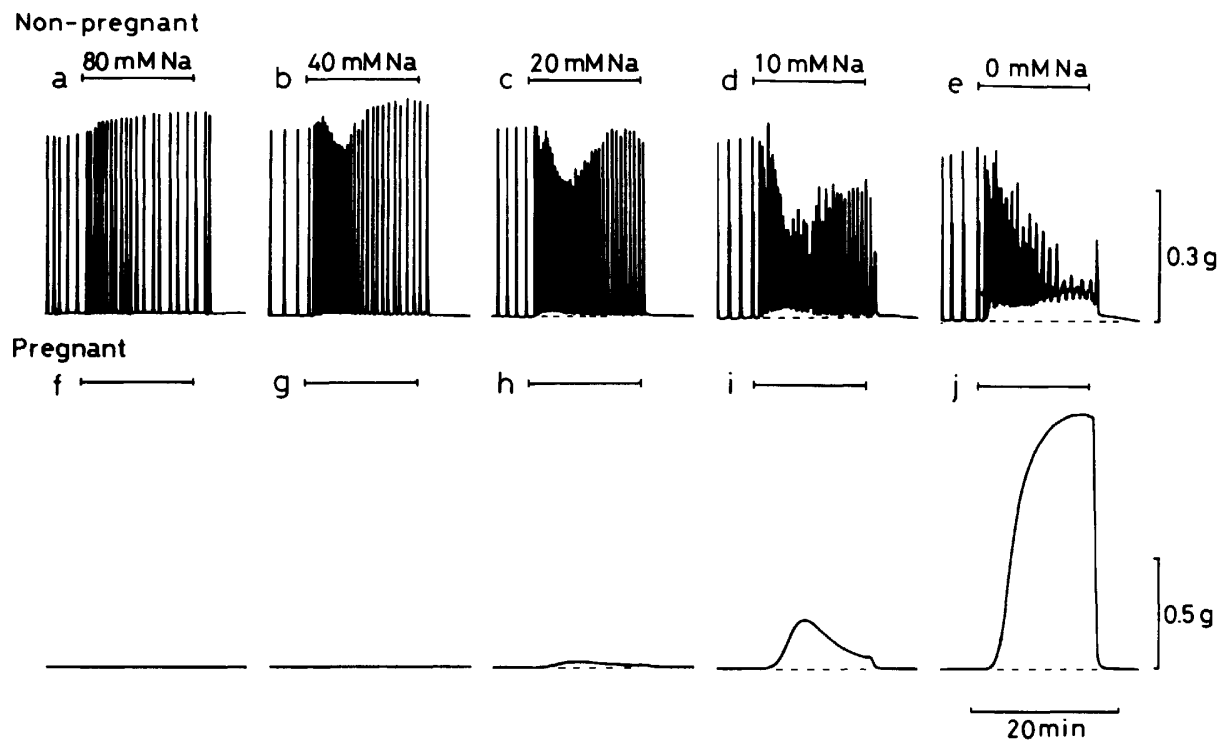

Fig. 1. Effects of reducing external Na concentrations on the mechanical activity in the circular muscles of non-pregnant (oestrus, a-e) and pregnant (the 18th day, $f-j$ ) myometria. Na was replaced isosmotically with Tris. Note that the tonic contraction produced by $\mathrm{Na}$ removal was much larger in the pregnant than the nonpregnant myometrium, although the size of preparations was nearly the same.

mI.U./ml, Sigma) in the same preparations. The maximum tension of oxytocininduced contractions was about $20 \%$ larger, but more stable, than that of spontaneous contractions.

\section{RESULTS}

\section{Effects of Na-removal}

In the non-pregnant rat myometrium, both circular and longitudinal muscle strips showed the spontaneous phasic contractions, whose pattern of activity varied from preparation to preparation. On the other hand, the muscle strips taken from the pregnant myometrium (the 12th-20th day of pregnancy) had spontaneous activity of a low frequency and some preparations were quiescent in both layers.

Figure 1 shows effects of reducing the external $\mathrm{Na}$ concentrations $\left([\mathrm{Na}]_{\mathrm{o}}\right)$ for $15 \mathrm{~min}$ on the circular muscles of the non-pregnant (Fig. la-e) and pregnant (the 18th day) myometria (Fig. If-j). In the non-pregnant muscle, the reduction of $[\mathrm{Na}]_{\mathrm{o}}$ increased the frequency of the spontaneous contractions, and at the $\mathrm{Na}$ concentrations below $20 \mathrm{~mm}$ the basal tone was increased and the peak tension of phasic contractions decreased (Fig. 1c-e), confirming the previous observation (Masahashi and Tomita, 1983). The tonic component of contraction induced by 


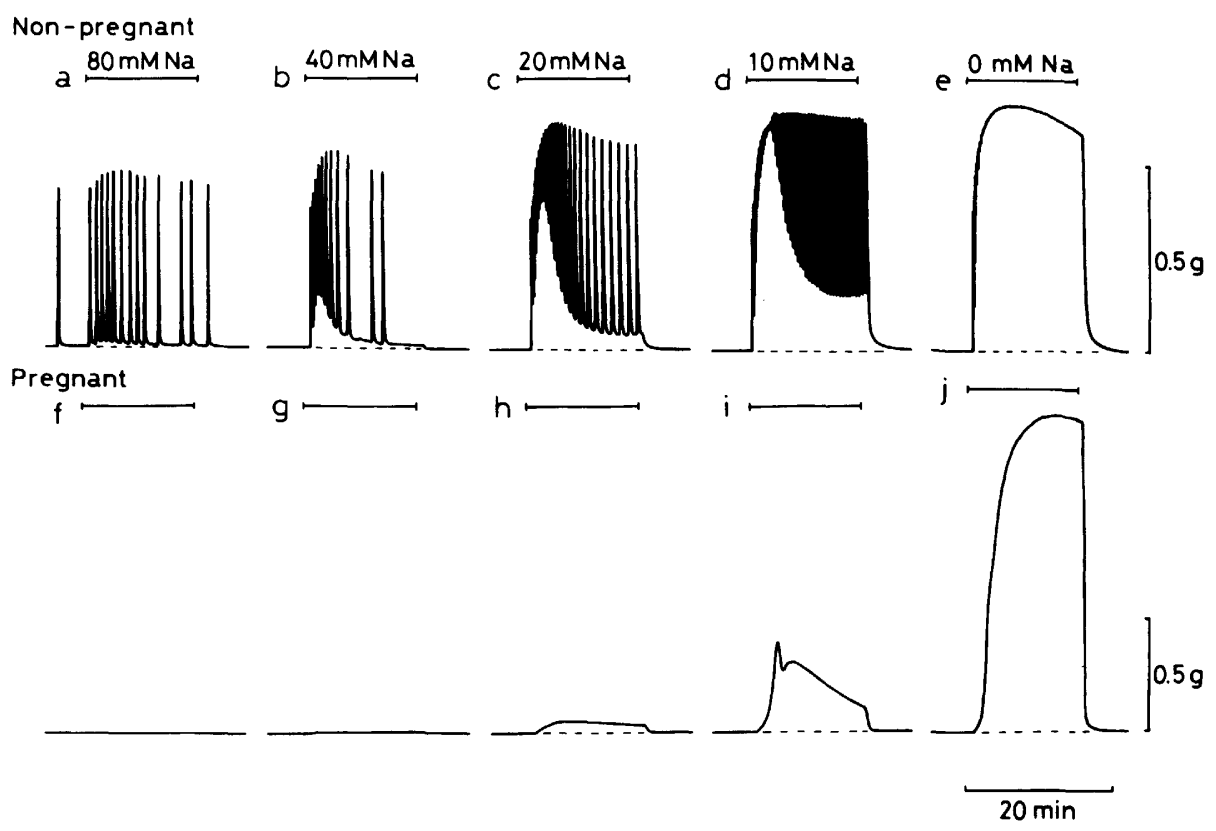

Fig. 2. Effects of reducing external Na concentrations on the longitudinal muscles of the same non-pregnant $(a-e)$ and pregnant $(f-j)$ rats as used for Fig. 1. Note that complete removal of $\mathrm{Na}$ produced a similar response both in non-pregnant and pregnant myometria.

Na-removal was only $10-20 \%$ of the spontenous phasic contractions. In the circular muscle obtained from pregnant rats the tonic contraction appeared when $[\mathrm{Na}]_{\mathrm{o}}$ was reduced to less than $20 \mathrm{~mm}$ (Fig. 1h, i) and this became very large on complete removal of $\mathrm{Na}$ (Fig. 1j).

In preganant circular muscles which had a spontaneous mechanical activity, reduction of $[\mathrm{Na}]_{0}$ first decreased the phasic contraction in size without changing its frequency, but it was reduced or abolished as the tonic contraction was developed in $\mathrm{Na}$-deficient solution. The maximum tonic tension developed in $\mathrm{Na}$-free solution was larger than the peak tension of spontaneous contractions or that of phasic contractions induced by oxytocin $(10 \mathrm{mI} . \mathrm{U} . / \mathrm{ml})$.

Although the degree and the pattern of the response to Na-removal $(0-\mathrm{Na}$ contraction) varied in different circular preparations, there were clear tendencies that non-pregnant preparations were more susceptible to reduction of $[\mathrm{Na}]_{0}$ than pregnant preparations, but that the tonic contraction in Na-free solution was much larger in pregnant than non-pregnant muscles of a similar size. The difference between $\mathrm{Na}$-free contractions in non-pregnant and pregnant myometria was also very clear when compared with the phasic contractions produced spontaneously or by oxytocin.

A typical example of the similar experiments on the longitudinal muscle is 


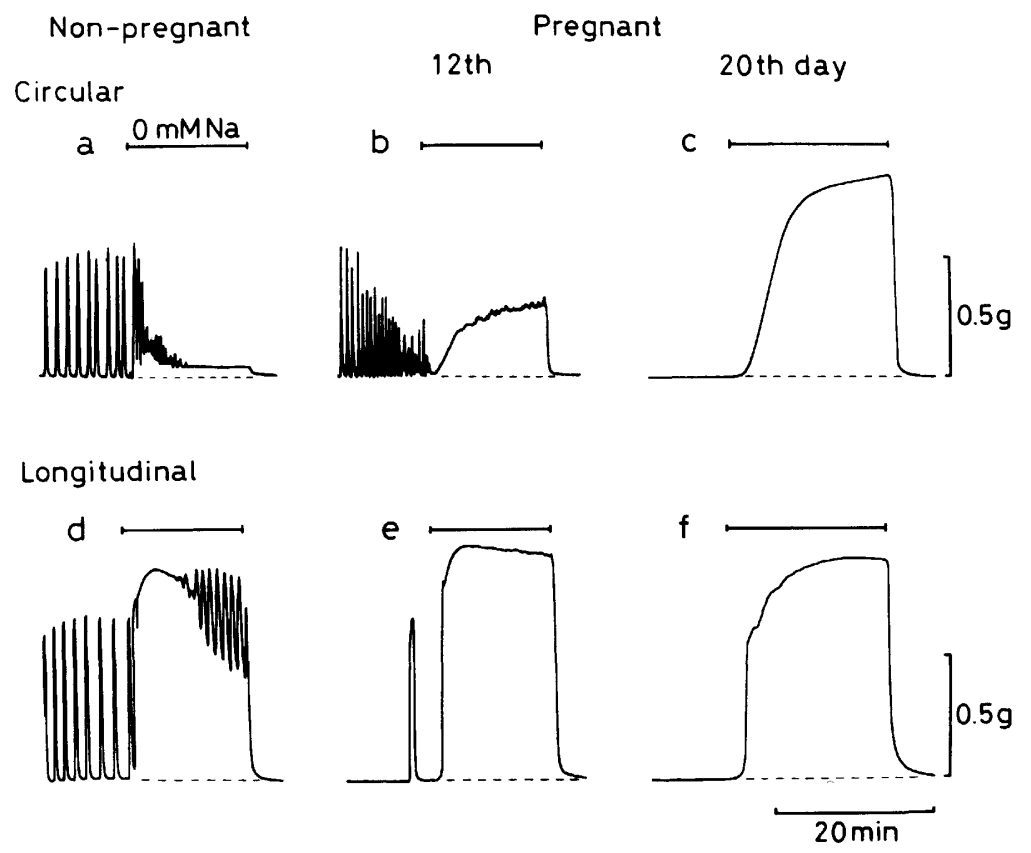

Fig. 3. Comparison of the 0-Na contractures between circular (upper) and longitudinal muscles (lower) of non-pregnant (a,d), and pregnant rats (b, e: the 12th day; and $c, f$ : the 20th day. Note that during pregnancy, the response to Na-removal changed markedly in the circular muscle compared with the longitudinal muscle.

shown in Fig. 2. In the non-pregnant myometrium (Fig. 2a-e), the increase in tonic component with lowering $[\mathrm{Na}]_{\mathrm{o}}$ was greater in the longitudinal muscle than in the circular muscle, as previously shown (MASAHASHI and TomiTA, 1983). When Na was removed completely, the maximum tonic contraction was larger than the spontaneous phasic contraction and its maximum was reached in 5-10 min. The response to Na-removal in the longitudinal muscle of pregnant myometrium was essentially the same as that of non-pregnant myometrium (Fig. $2 \mathrm{f}-\mathrm{j}$ ). Thus, in the pregnant myometrium, muscle strips of both layers produced a large tonic contraction in $\mathrm{Na}$ free solution, although the rate of tension development was slower in the pregnant than in the non-pregnant myometrium.

\section{Changes in 0-Na contraction during pregnancy}

Figure 3 shows the responses to Na-removal in non-pregnant (Fig. 3a, d), midpregnant (Fig. 3b, e: the 12th day), and late pregnant (Fig. 3c, f: the 20th day of pregnancy) preparations. In the circular muscle, the tonic tension produced by Naremoval was markedly increased during pregnancy. On the other hand, in the longitudinal muscle, no remarkable change in the pattern of tension development 


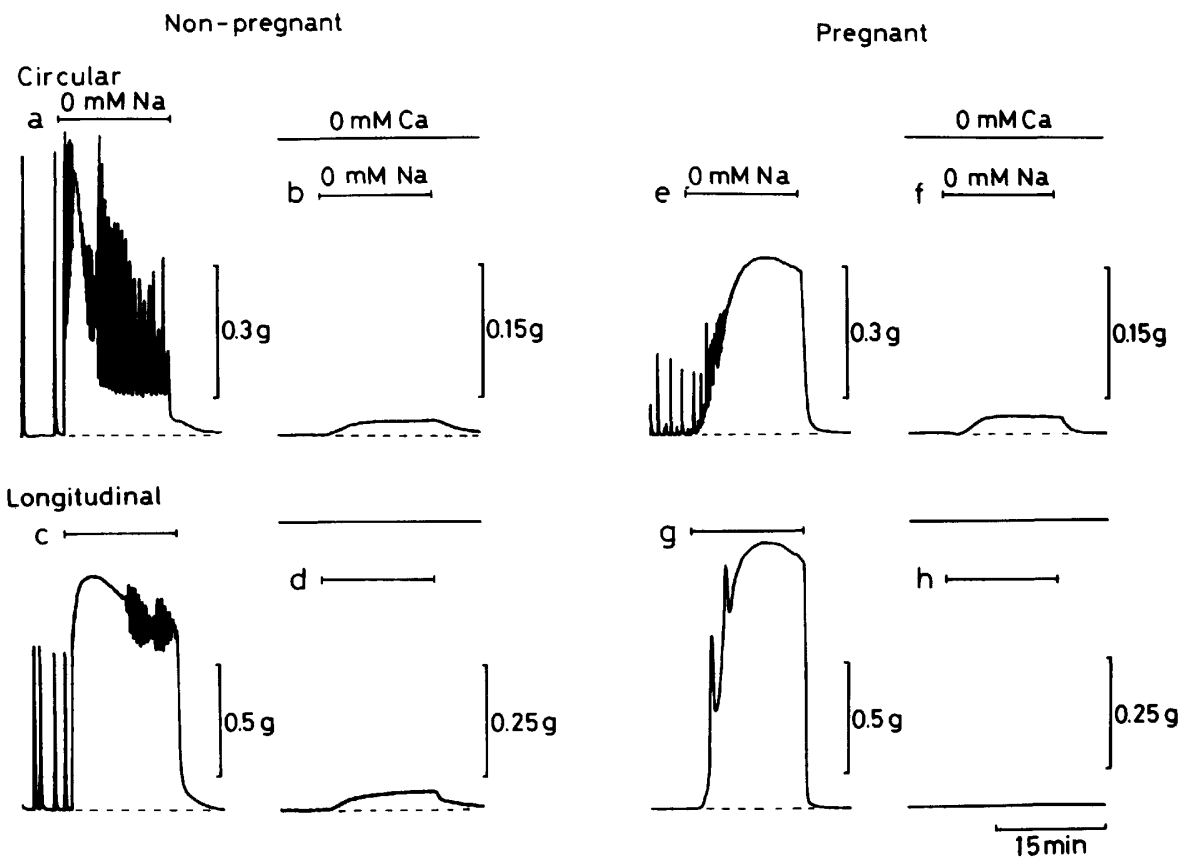

Fig. 4. Small contraction produced by Na-removal in Ca-free solution containing $0.5 \mathrm{~mm}$ EGTA, in circular (upper) and longitudinal (lower) muscles of non-pregnant (a-d) and pregnant (the 20th day, e-h) myometria. After taking control responses to Na-removal from normal solution (a, c,e,g), the preparations were exposed to $\mathrm{Ca}$-free solution; $30 \mathrm{~min}$ later, $\mathrm{Na}$ was removed for $15 \mathrm{~min}(\mathrm{~b}, \mathrm{~d}, \mathrm{f}, \mathrm{h})$. Note no response to Na-removal in pregnant longitudinal muscle exposed to $\mathrm{Ca}$-free solution.

was observed. In mid-pregnancy, both circular and longitudinal muscles showed spontaneous phasic contractions. With the progress of pregnancy, the number of spontaneously active strips was decreased, particularly in the longitudinal layer. In the circular muscles, the size of tonic contraction produced by Na-removal relative to that of the spontaneous contraction was about $50 \%$ in mid-pregnancy and $150 \%$ in late pregnancy. In the longitudinal muscle, the tonic tension in $\mathrm{Na}$-free solution was between $100-150 \%$ of spontaneous contraction and this ratio remained roughly constant during pregnancy.

\section{Response to Na-removal in Ca-free solution}

It has been noticed in the non-pregnant myometrium that the removal of the external $\mathrm{Na}$ after preincubation in $\mathrm{Ca}$-free solution produced a small sustained contraction in both circular and longitudinal muscles (MASAHASHI and TOMITA, 1983). This was confirmed in the present experiments, as shown in Fig. 4. In the pregnant myometrium, only the circular muscle produced such a response (Fig. 4f), 


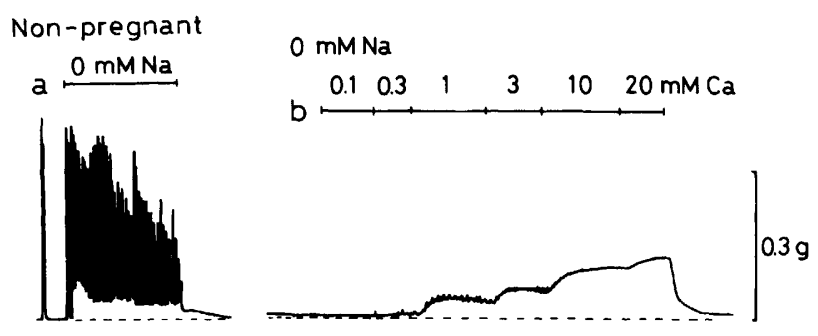

A

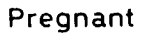

c $\stackrel{0 \mathrm{mM} \mathrm{Na}}{\longleftarrow}$
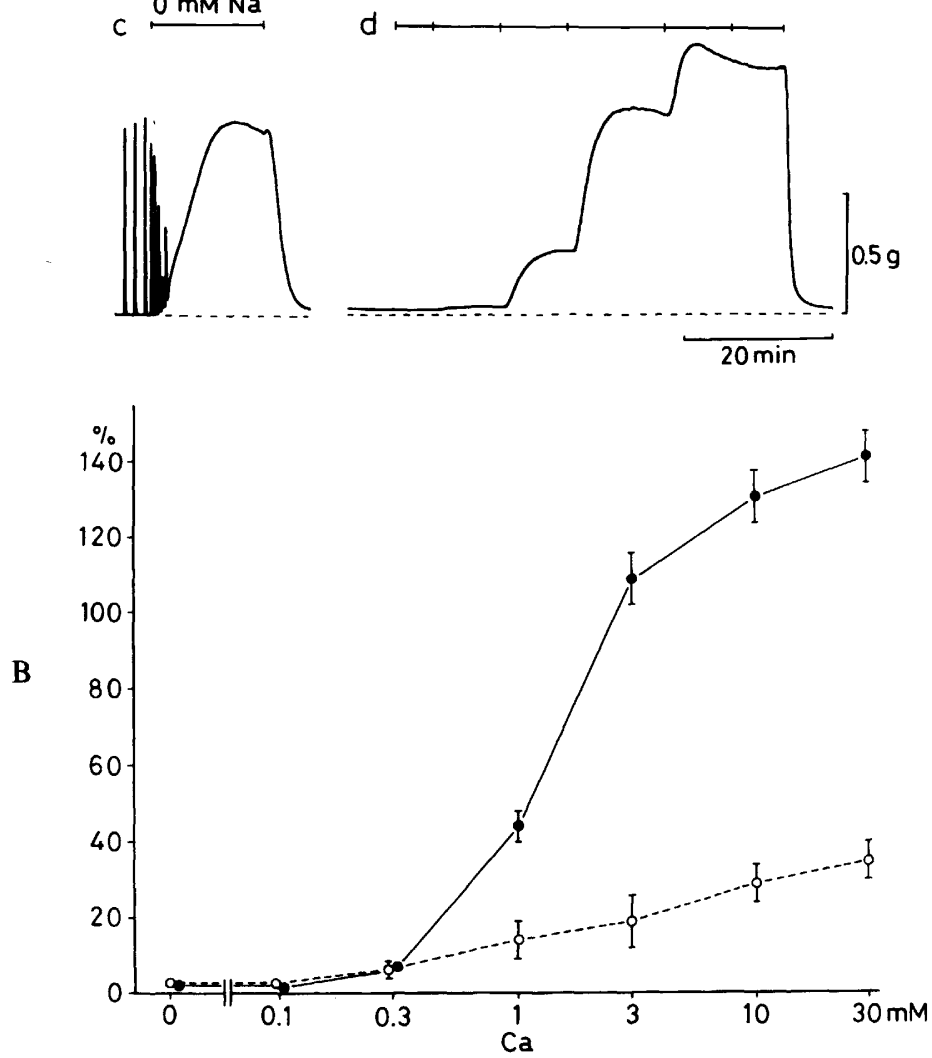

Fig. 5. A: responses to $\mathrm{Ca}$ readmission in the absence of $\mathrm{Na}$ in the circular muscles of non-pregnant (upper) and pregnant (the 19th day) myometria (lower). a and c, control responses to Na-removal from normal solution. $b$ and $d$, responses to cumulative application of $\mathrm{Ca}$ (as shown on the top), after exposure to $\mathrm{Na}$-free solution for $20 \mathrm{~min}$. See text for further explanation. B: concentration-tension relationship for $\mathrm{Ca}$ in the circular muscles exposed to $\mathrm{Na}$-free solution, as plotted based on the experiments shown in A. Open circles, non-pregnant $(n=6)$, and filled circles, pregnant (the 17th-20th day, $n=10$ ). Tension development (ordinate) was expressed relative to phasic contractions evoked by oxytocin $(10 \mathrm{mI} . \mathrm{U} . / \mathrm{ml})$ in normal solution. 


\section{Non-pregnant}

$0 \mathrm{mM} \mathrm{Na}$

A

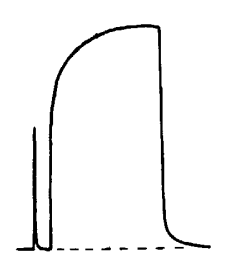

Pregnant

c
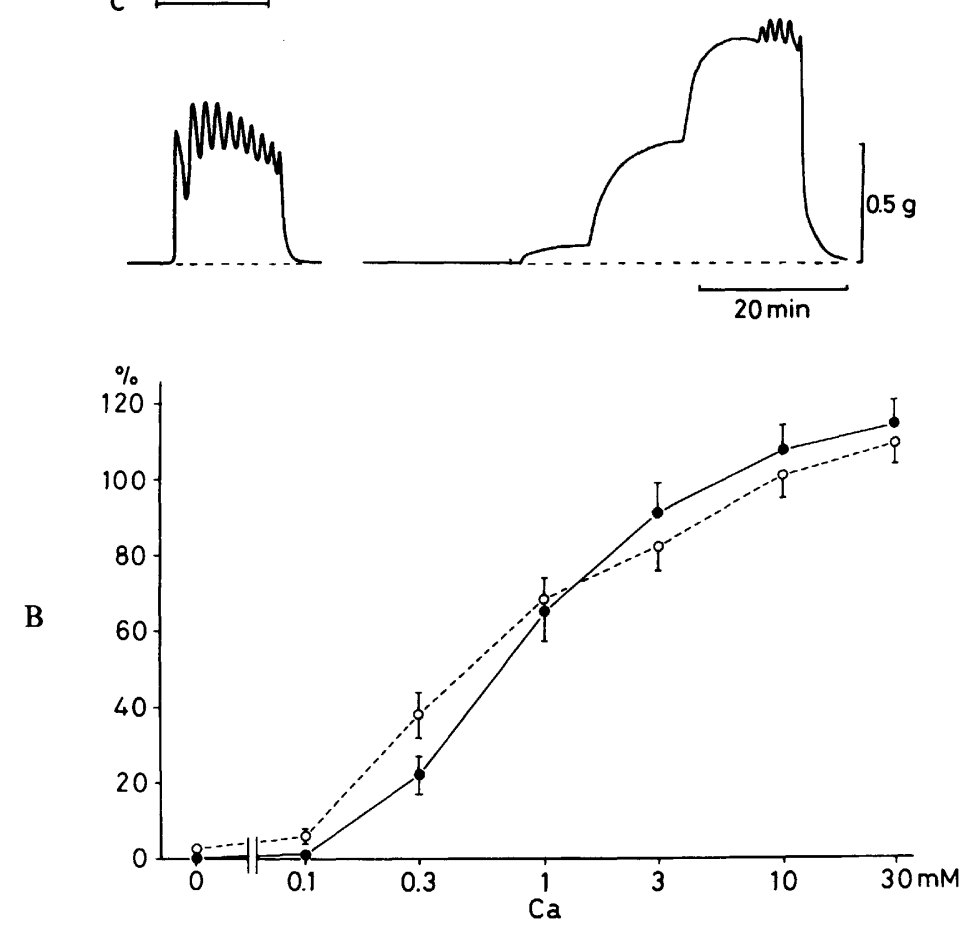

Fig. 6. Experiments in the longitudinal muscles similar to those shown in Fig. 5. A: tension response in non-pregnant (upper) and pregnant (the 19th day) muscles (lower). B: concentration-tension curves for $\mathrm{Ca}$ in the longitudinal muscle of nonpregnant (open circles, $n=10$ ) and pregnant myometria (closed circles, the 17 th20 th day, $n=10$ ).

and the longitudinal muscle failed to produce any clear increase in tension by $\mathrm{Na}-$ removal (Fig. 4h). Except for the circular muscle of pregnant myometrium, the increase in tension by Na-removal could be repeatedly produced many times without any reduction during a prolonged exposure to $\mathrm{Ca}$-free solution containing 


\section{$0.5 \mathrm{~mm}$ EGTA.}

\section{Ca-induced tension in Na-free solution}

After removing both $\mathrm{Na}$ and $\mathrm{Ca}$ from the external medium, $\mathrm{Ca}$ was readmitted cumulatively in the absence of $\mathrm{Na}$. An example of such experiments on the circular muscles from non-pregnant (Fig. 5A-a, b) and pregnant (Fig. 5A-c, d) myometria is shown in Fig. 5A. In the non-pregnant myometrium, the tension started to develop at $0.3 \mathrm{~mm} \mathrm{Ca}$ and reached nearly the maximum at $20 \mathrm{~mm}$ (Fig. 5A-b). The maximum tension was much smaller than the spontaneous contractions in normal solution containing $2.4 \mathrm{~mm} \mathrm{Ca}$ (Fig. 5A-a). In the pregnant myometrium, a clear tension development was also observed at $0.3 \mathrm{~mm} \mathrm{Ca}$ and the maximum reached, at $10 \mathrm{~mm}$ $\mathrm{Ca}$ in this preparation, was larger than the spontaneous contraction (Fig. 5A-d).

Figure 5B shows the concentration-tension relationship for $\mathrm{Ca}$ in the circular muscles obtained from non-pregnant $(n=6)$ and pregnant myometrium $(n=10)$. The tension is expressed as a percentage of the phasic contraction evoked by oxytocin $(10 \mathrm{mI} . \mathrm{U} . / \mathrm{ml})$ in normal solution. There was no difference in tension development below $0.3 \mathrm{~mm}$ Ca between non-pregnant and pregnant myometria, but beyond this concentration, pregnant myometrium produced much larger tension responses.

Experiments similar to those shown in Fig. 5 were also carried out on longitudinal muscles. As shown in Fig. 6A and B, the non-pregnent muscle was more sensitive to $\mathrm{Ca}$ than the pregnant muscle, but the tension development at higher $\mathrm{Ca}$ concentrations was similar for both myometria.

When the same concentration of $\mathrm{Ca}$ was repeatedly applied for $10 \mathrm{~min}$ intervals in $\mathrm{Na}$-free solution, the tension development became gradually smaller. This reduction was recovered by treating the preparation with normal solution containing $122 \mathrm{~mm} \mathrm{Na}$, suggesting that a decrease in intracellular $\mathrm{Na}$ concentration has some inhibitory effect on the tension, as previously reported for the taenia of guinea-pig caecum (BRADING et al., 1980). Thus, cumulative application of Ca during a prolonged exposure to $\mathrm{Na}$-free solution might have reduced the steepness of concentration-tension curve for $\mathrm{Ca}$.

\section{Effects of excess $K$ solution}

In all preparations examined (circular and longitudinal muscles in nonpregnant and pregnant myometria), the concentration-tension relationship for $\mathrm{Ca}$ in $\mathrm{Na}$-free solution was not essentially affected by increasing the external $\mathrm{K}$ concentration from 5.9 to $40 \mathrm{~mm}$, although there was a tendency that tension development was slightly larger in excess $\mathrm{K}$ medium, particularly in the circular muscle.

Figure 7 shows comparison of contractions evoked by simple Na-removal, combination of Na-removal and $40 \mathrm{~mm} \mathrm{~K}$, and $40 \mathrm{~mm} \mathrm{~K}$ only, in the presence of $2.4 \mathrm{~mm} \mathrm{Ca}$. In the non-pregnant circular muscle, the tonic response to Na-removal was very similar to that caused by $40 \mathrm{~mm} \mathrm{~K}$, except that rhythmic phasic contractions often superimposed on top of the tonic component of $0-\mathrm{Na}$ con- 


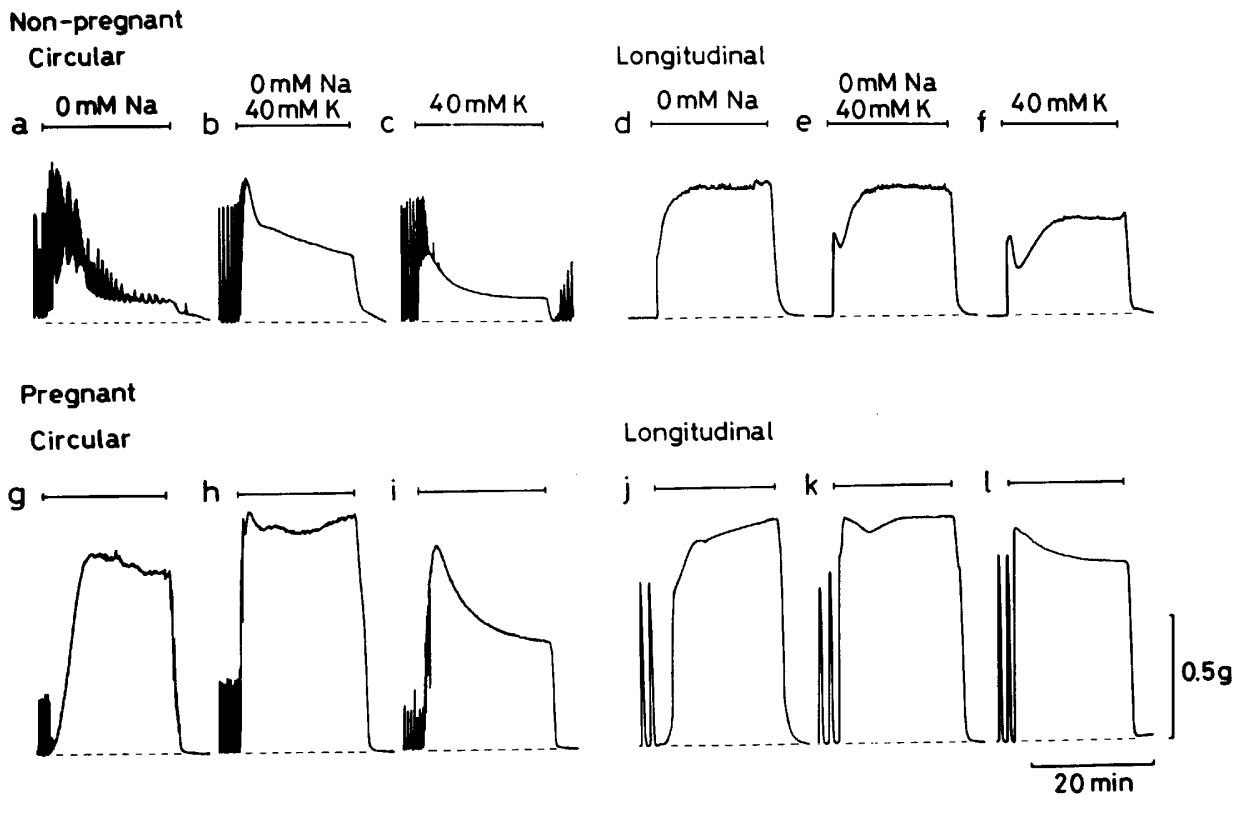

Fig. 7. Responses to Na-free ( $5.9 \mathrm{~mm} \mathrm{~K})$, Na-free ( $40 \mathrm{~mm} \mathrm{~K})$, and $40 \mathrm{~mm} \mathrm{~K}(87.4 \mathrm{~mm}$ $\mathrm{Na}$ ) solutions in the presence of $2.4 \mathrm{mM} \mathrm{Ca}$, taken at 30 -min intervals. a-c, nonpregnant circular; $d-f$, longitudinal muscle; and $g-i$, pregnant (the 18th day) circular; $\mathrm{j}-\mathrm{l}$, longitudinal muscle. Calibration for tension is applied only to $\mathrm{d}-\mathrm{l}$; and for $\mathrm{a}-\mathrm{c}$, it is $0.3 \mathrm{~g}$.

traction, but not of K-contracture. However, in other muscles, K-contracture was generally smaller than the contraction caused by simple $\mathrm{Na}$-removal. When $\mathrm{Na}$ was completely removed from $40 \mathrm{mM} \mathrm{K}$ solution the tension became larger than that evoked by $40 \mathrm{~mm} \mathrm{~K}$ solution containing normal Na concentration (122 mM).

\section{DISCUSSION}

In the non-pregnant myometrium, the maximum tension developed in spontaneous contractions is comparable both in longitudinal and circular muscles, when expressed in a cross-sectional unit area (IZUMI, 1985). The tension is increased during gestation in both muscle layers, although the increase is greater in longitudinal than circular muscle. On the other hand, the tonic contraction produced by $\mathrm{Na}$-removal ( 0 - $\mathrm{Na}$ contraction) is much smaller in the circular muscle than in the longitudinal muscle of non-pregnant myometrium, as previously reported (MASAHASHI and TOMITA, 1983). During pregnancy, the 0-Na contraction increases in the circular muscle, whereas that in the longitudinal muscle remains more or less the same. The concentration-tension relationship for $\mathrm{Ca}$ obtained in Na-free solution indicates that the main change in the circular muscle during 
pregnancy is not the sensitivity to $\mathrm{Ca}$ but an increase in tension development at higher $\mathrm{Ca}$ concentrations. Another change is that the sensitivity to $\mathrm{Ca}$ is slightly, but significantly, decreased in the longitudinal muscle during pregnancy. The low sensitivity to $\mathrm{Ca}$ in the longitudinal muscle of pregnant myometrium seems to be related to lack of the contraction independent of the external $\mathrm{Ca}$. The oxytocininduced tonic contraction is known to be produced during a prolonged exposure to Ca-free solution in the longitudinal muscle of non-pregnant rat myometrium (SAKAI et al., 1981, 1982; AsHOORI et al., 1985). A similar type of contraction is produced by Na-removal in both layers of non-pregnant rat myometrium (MASAHASHI and ToMITA, 1983), and also in the circular, but not longitudinal, muscle of rat pregnant myometrium.

The alteration of electrical properties during pregnancy has also been found mainly in the circular muscle (OSA and KatASE, 1975; Osa and KaWARABAYASHI, 1977; OSA and FuJino, 1978; ANDERSON et al., 1981; KishIKAWA, 1981). In the nonpregnant myometrium, the burst of spike activity appears on slow depolarization of the membrane. However, in the circular muscle the slow depolarization forms a prominent plateau superimposed with a few spikes (the plateau type), while in the longitudinal muscle a train of spikes is accompanied with a small sustained depolarization (the spike type). In the circular muscle of pregnant myometrium, the plateau type can still be observed until the 13-15th day of pregnancy, but when approaching near term, the action potential changes to the spike type, so that the pattern of electrical activity becomes similar both in circular and longitudinal layers in late pregnancy.

It is not clear whether this alteration of electrical activity underlies the changes in mechanical responses to Na-removal during pregnancy observed in the present experiments. Repetitive electrical stimulation produces maintained contraction in the longitudinal muscle, but it fails to produce summation of contractions in the circular muscle in mid-pregnancy (the 10th-15th day) (OsA and KATASE, 1975). However, at near term, the circular muscle becomes responsive to repetitive stimulation with sustained contraction as in the longitudinal muscle; this is probably related to alteration of electrical activity. Therefore, it may be argued that the change in membrane properties is somehow related to modification of the 0 -Na contraction by pregnancy. However, alteration of depolarization-contraction coupling may also be involved.

It has been shown that pregnant mouse myometrium (likely to be longitudinal muscle) is depolarized on Na-removal (OsA, 1971, 1973). Similar depolarization has also been confirmed in the longitudinal muscle of non-pregnant rat myometrium (TомітA et al., 1984). The small 0 - $\mathrm{Na}$ contraction in the circular muscle of nonpregnant myometrium may be explained by assuming smaller depolarization or poorer synchronization of spike activity in $\mathrm{Na}$-free solution compared with that in the longitudinal muscle or in the circular muscle of pregnant myometrium. Although electrophysiological studies have to be done to examine this possibility, it is, however, unlikely that the difference in depolarization or spike activity of the 
membrane is responsible for the difference in mechanical response to Na-removal. In the circular muscle of non-pregnant myometrium, the $\mathrm{K}$ contracture induced by $40 \mathrm{~mm} \mathrm{~K}$ is much smaller than spontaneous contractions, as is found for the $0-\mathrm{Na}$ contraction. Therefore, the small $0-\mathrm{Na}$ contraction in this muscle is probably not due to a small degree of depolarization or a poor synchronization of activity, but due to a limited $\mathrm{Ca}$ influx or a low efficiency of the excitation-contraction coupling for the sustained contraction compared with phasic contractions.

We are grateful to Professor Y. Tomoda for his continuous encouragement in this experiment.

\section{REFERENCES}

Anderson, G. F., Kawarabayashi, T., and Marshall, J. M. (1981) Effect of indomethacin and aspirin on uterine activity in pregnant rats: Comparison of circular and longitudinal muscle. Biol. Reprod., 24: 359-372.

Angles D'Auriac, G. and Worcel, M. (1976) Cellular levels of cAMP and cGMP in rat uterus smooth muscle. Effects of angiotensin, carbachol and various metabolic conditions. In: Smooth Muscle Pharmacology and Physiology, ed. by Worcel, M. and VASSORT, G., INSERM, Paris, pp. 101-111.

Ashoori, F., TAKAI, A., and TOMita, T. (1985) The response of non-pregnant rat myometrium to oxytocin in Ca-free solution. Br. J. Pharmacol., 84: 175-183.

Brading, A. F., BurnetT, M., and Sneddon, P. (1980) The effect of sodium removal on the contractile responses of the guinea-pig taenia coli to carbachol. J. Physiol. (Lond.), 306: 411-429.

IzUMI, H. (1985) Changes in the mechanical properties of the longitudinal and circular muscle tissues of the rat myometrium during gestation. Br. J. Pharmacol., 86: 247-257.

KISHIKAWA, T. (1981) Alterations in the properties of the rat myometrium during gestation and post partum. Jpn. J. Physiol., 31: 515-536.

MA, T. S. and Bose, D. (1977) Sodium in smooth muscle relaxation. Am. J. Physiol., 232: C59-C66.

Marshall, J. M. (1963) Behavior of uterine muscle in $\mathrm{Na}^{+}$-deficient solutions: Effects of oxytocin. Am. J. Physiol., 204: 732-738.

Masahashi, T. and Tomita, T. (1983) The contracture produced by sodium removal in the non-pregnant rat myometrium. J. Physiol. (Lond.), 334: 351-363.

OsA, T. (1971) Effect of removing the external sodium on the electrical and mechanical activities of the pregnant mouse myometrium. Jpn. J. Physiol., 21: 607-625.

OsA, T. (1973) The effect of sodium, calcium and manganese on the electrical and mechanical activities of the myometrial smooth muscle of pregnant mice. Jpn. J. Physiol., 23: 113-133.

OsA, T. and Fujino, T. (1978) Electrophysiological comparison between the longitudinal and circular muscles of rat uterus during the estrous cycle and pregnancy. Jpn. J. Physiol., 28: 197-209.

Osa, T. and KATASE, T. (1975) Physiological comparison of the longitudinal and circular muscle of the pregnant rat uterus. Jpn. J. Physiol., 25: 153-164.

Osa, T. and Kawarabayashi, T. (1977) Effects of ions and drugs on the plateau potential in the circular muscle of pregnant rat myometrium. Jpn. J. Physiol., 27: 111-121.

SaKaI, K., Higuchi, K., YamaguChI, T., and UChida, M. (1982) Oxytocin-induced Ca-free 
contraction of rat uterine smooth muscle: Effects of preincubation with EGTA and drugs. Gen. Pharmacol., 13: 393-396.

SaKaI, K., Yamaguchi, T., and UCHIDA, M. (1981) Oxytocin-induced Ca-free contraction of rat uterine smooth muscle: Effects of divalent cations and drugs. Arch. Int. Pharmacodyn., 250: 40-54.

Tomita, T., ToKuno, H., and Masahashi, T. (1984) Effects of Na removal in smooth muscles. Jikeikai Med. J., 30 (Suppl. 1): 89-96. 